\title{
EPMA on Copper-Gold Alloys - A CCQM/SAWG Pilot Study
}

\author{
V.-D. Hodoroaba,* R.B. Marinenko,** J.A. Small** and W.E.S. Unger* \\ * BAM Federal Institute for Materials Research and Testing, 12200 Berlin, Germany \\ ** NIST, National Institute of Standards and Technology, Gaithersburg, MD 20899
}

CCQM (Consultative Committee for Amount of Substance - Metrology in Chemistry) is an international body of the Meter Convention (BIPM) established as a framework of National Metrology Institutes running inter-laboratory of comparisons to demonstrate the international comparability of chemical measurements, with traceability to international or national reference standards. In 2003 the surface analysis working group (SAWG) was established at CCQM and since then several inter-laboratory comparisons in the field of EPMA have been carried out. In many cases the EPMA results deviated significantly from each other and the expanded uncertainties were greater than expected. Both methods EDS and WDS were employed. However, light elements such as carbon and nitrogen were the major elements analyzed and it is known that the corresponding lowenergy characteristic X-ray lines are more challenging to quantify by EPMA than the higher energy lines of elements with higher atomic numbers. Moreover, the standards selected and their qualities were variable, the quantification models were different, and the acquisition parameters were not specified sufficiently in the analysis instructions.

A new pilot study (CCQM-P130) on binary metal alloys containing copper and gold has been initiated under the leadership of BAM and NIST. The complete set of four specimens and two pure reference standards constitute the NIST SRM 482 for microanalysis. The high energy $\mathrm{Cu}-\mathrm{K}$ and $\mathrm{Au}-$ L lines at about $8 \mathrm{keV}$ and $10 \mathrm{keV}$, respectively, will be used for quantification, but also the more challenging $\mathrm{Cu}-\mathrm{L}$ and $\mathrm{Cu}-\mathrm{M}$ lines with energies below $2.5 \mathrm{keV}$ can be used for analysis in the lowenergy range. The pure copper and gold reference standards will also be supplied to all participants in the pilot study. Participants are asked to report the determined k-values as the primary measurand. In order to be able to evaluate also the different uncertainty budgets associated with the matrix correction procedures and various attenuation coefficients used by each participant, the elemental concentrations are also asked as a second (derived) measurand. Also the acquired X-ray spectra in emsa-format are asked from the ED-EPMA participants. This is necessary to enable the evaluation of the uncertain-ties of the background subtraction with a single procedure.

The challenging but most interesting part of the pilot study is the evaluation of the uncertainty budget. Procedures to determine the uncertainty budget are given according to the ISO Guide to the Expression of Uncertainty in Measurement (GUM), i.e. type A (statistical) and type B (expert based estimation of systematic errors or biases) $[1,2]$.

\section{References}

[1] JCGM 100:2008 GUM 1995 with minor corrections, Evaluation of measurement data - Guide to the expression of uncertainty in measurement (2008)

[2] R. B. Marinenko, S. Leigh, 11th European Workshop on Modern Developments and Applications in Microbeam Analysis IOP Publishing, IOP Conf. Series: Materials Science and Engineering 7 (2010) 012017. 


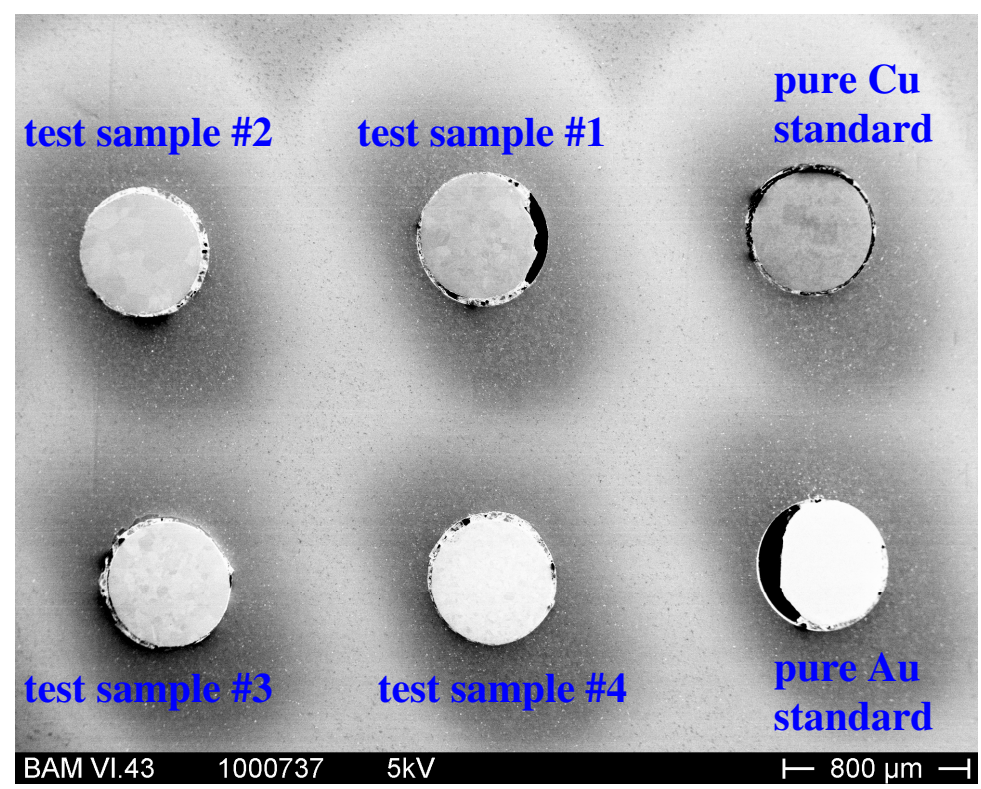

FIG. 1. SEM micrograph showing the surface of the prepared set of six specimens mounted in an aluminum block: the four specimens to be analyzed and the two reference standards.
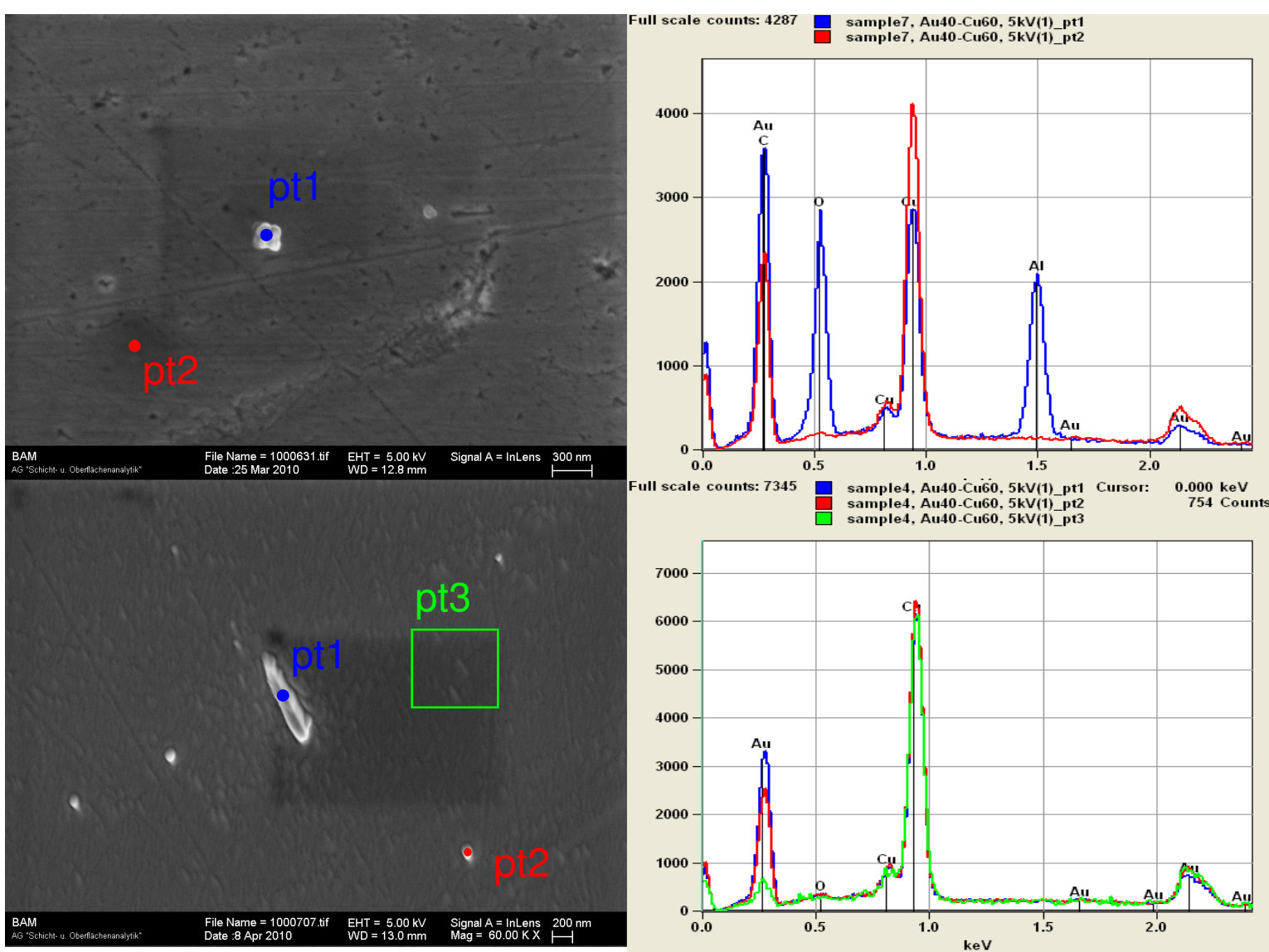

FIG. 2. Top: SEM micrograph of the specimen surface after grinding and polishing; the point EDS analysis (blue) indicates presence of spurious aluminum oxide; Bottom: SEM micrograph and EDS spectra taken from the specimen surface after Ar sputtering indicating no significant surface contamination. 